\title{
Quantum backflow across a black hole horizon in a toy model approach
}

\author{
Dripto Biswas $\odot^{*}$ \\ School of Physical Sciences, National Institute of Science Education and Research, \\ Bhuabneswar, Odisha 752050, India \\ Subir Ghosh $\odot$ \\ Physics and Applied Mathematics Unit, Indian Statistical Institute, Kolkata, West Bengal 700108, India
}

(Received 28 May 2021; accepted 13 October 2021; published 24 November 2021)

\begin{abstract}
Quantum backflow, discovered quite a few years back, is a generic purely quantum phenomenon, in which the probability of finding a particle in a direction is nonzero (and increasing for a certain period of time) even when the particle has with certainty a velocity in the opposite direction. In this paper, we study quantum backflow of a quantum particle across the event horizon of a Schwarzschild black hole. In a toy model approach, we consider a superposition of two ingoing solutions and observe the probability density and probability current. We explicitly demonstrate a nonvanishing quantum back flow in a small region around the event horizon. This is in contrast to the classical black hole picture, that once an excitation crosses the horizon, it is lost forever from the outside world. Deeper implications of this phenomenon are speculated. We also study quantum backflow for another spacetime with horizon, the Rindler spacetime, where the phenomenon can be studied only within the Rindler wedge.
\end{abstract}

DOI: 10.1103/PhysRevD.104.104061

\section{INTRODUCTION}

Consider a particle moving along, say, the $x$ axis having with certainty a positive velocity along the right-hand direction with respect to some arbitrary origin $x=0$. For a classical particle obviously the chance that it will be detected at $x>0$ will increase with time. However, for a quantum particle, on the contrary, for certain wave functions, the probability of the particle's position at $x<0$ actually increases with time, for a finite period of time. This generic counterintuitive phenomenon is the well known quantum backflow (QBF). It can occur for a free particle or one in presence of a potential. Even if the particle's wave function is centered around $x=0$ and is constructed out of only positive momenta, it can still possess nonvanishing probability of being observed at $x<0$ and the probability can even increase for finite periods of time. Thus, the quantum-mechanical current at the origin can be negative with the probability flowing in opposite direction to the momenta. In this paper, for the first time, we study QBF of ingoing modes crossing the black hole $(\mathrm{BH})$ event horizon. Obviously this is contrary to conventional $\mathrm{BH}$ physics

\footnotetext{
*dripto.biswas@niser.ac.in
}

Published by the American Physical Society under the terms of the Creative Commons Attribution 4.0 International license. Further distribution of this work must maintain attribution to the author(s) and the published article's title, journal citation, and DOI. Funded by SCOAP . wisdom that nothing can come out of the $\mathrm{BH}$ horizon. It can have deep impact in fundamental $\mathrm{BH}$ physics. It needs to be mentioned that QBF is a generic quantum phenomenon and in the QBF effects studied so far (see Refs. [1-21]) the presence of a spacetime horizon (as in $\mathrm{BH}$ ) does not arise.

QBF was revealed by Allcock's work [1] on the arrival time problem in quantum theory (see also [2,3]). It also appeared in the time operator construction in the Heisenberg time-energy uncertainty relation [4] and decay of quasistable quantum systems where the small inward current was interpreted as QBF [5]. Bracken and Melloy [6] showed that, (i) the total amount of QBF is bounded (by a dimensionless number computed numerically $\approx 0.04$ ) and (ii) the bound has a universal character, independent of the time duration, particle mass and $\hbar$. A subtle limiting procedure proves vanishing of the effect in classical framework [7]. A numerical study [8] revealed a structure of the wave function and the corresponding approximate analytic form of the wave function appeared in $[7,9,10]$ which yields a modest backflow. Its relativistic extension was studied in [11-13]. In our case, the BH horizon generates an effective potential and the impact of a linear potential was investigated in [13]. Connections between QBF and superoscillations was noted in [14]. A nonzero probability flux in classically forbidden regions, in the expansion of a wave packet in free space was discussed in [15]. (For a recent brief review see [10].) In a recent paper [16] direct evidence of QBF in an analog optical system has been reported. Other experimental proposals have been 
utilizing Bose-Einstein condensates [17,18], using solutions with both positive and negative momenta $[19,20]$ and a recent suggestion of moving the particle in a ring [21]. We now come to our main concern: possible relevance of QBF across a $\mathrm{BH}$ horizon.

In classical general relativity, nothing can come out of the region behind the event horizon of a $\mathrm{BH}$ (that surrounds the spacetime singularity) and reach an observer at a large distance. This idea was overthrown in a quantum framework whereby BHs can emit Hawking radiation which carries information about the mass, charge, and angular momentum of the BH. So far this is the only possible mechanism of information (in the form of only mass, angular momentum, and charge of the $\mathrm{BH}$ ) exchange between the $\mathrm{BH}$ and outside world. In this perspective QBF might have significance in providing another channel of information exchange between $\mathrm{BH}$ and outside observer. However, further discussion on this is outside the scope of the present work where we have established only the presence of QBF across $\mathrm{BH}$ horizon.

Hawking's seminal work $[22,23]$ showed that a BH can radiate with a characteristic (Hawking) temperature and ushered in $\mathrm{BH}$ thermodynamics, thus formalizing the $\mathrm{BH}$ area-entropy connection [24]. However, this originated the so-called information paradox-BH evaporation leaves only thermal radiation with a temperature that depends just on a few macroscopic BH parameters and no information about the BH constituents, transforming via nonunitary evolution, from a pure state to a mixed state. To keep unitarity intact, $\mathrm{BH}$ entropy has to follow the Page curve [25] and only recently it is becoming clear [26-28] how quantum entanglement can remove the information paradox. An alternative approach, for modelling a quantum $\mathrm{BH}$, advocated by t'Hooft $[29,30]$, hinges on applying unitary quantum mechanics at the $\mathrm{BH}$ horizon with the $\mathrm{BH}$ satisfying the Schrödinger equation. In this framework, scattering on the black hole horizon in a partial wave basis is studied in [31,32]. We, on the other hand, apply the Schrödinger equation to the particles on the BH horizon. In $[33,34]$, physical boundary conditions for the quantum particle wave equation at $\mathrm{BH}$ horizon reveal exponentially damped or enhanced solutions suggesting that particles instead of crossing the $\mathrm{BH}$ horizon are absorbed or reflected by it.

In this perspective, $\mathrm{QBF}$ across a $\mathrm{BH}$ horizon assumes significance, leading to open questions: can the backflow be directly interpreted as particles or at least can it transfer information across the horizon from the inside in some form of correlation between the ingoing wave and its QBF component? Although we will revisit this issue at the concluding section, we address a point that is bound to come up, i.e., is there any connection between $\mathrm{QBF}$ and the celebrated Hawking effect, leading to Hawking radiation. Let us just emphasize that these two are entirely different phenomena: the fundamental differences being that Hawking radiation is a quantum field theoretic phenomenon derived in a semiclassical framework where presence of the event horizon is crucial, whereas QBF is a quantum mechanical process and in the examples studied so far, the presence of an event horizon did not arise. The outcome of Hawking effect is that the radiation arises at the expense of the black hole mass and in the process the black hole might disappear completely. On the other hand, with the QBF we are looking at, where external particles crossing in to the black hole are considered and where part of this incoming flux can remain outside the horizon, and the question of black hole evaporation as a result of QBF does not arise. An underlying motivation of our work is the possibility that QBF might have some correlation with the major portion of ingoing flux.

We consider a simplified scenario, following earlier works [6], and study QBF pertaining to a superposition of two solutions of the Schrödinger equation, near the BH horizon. (We leave the more realistic problem of using wave packets for a future publication.) Since our model is time dependent but stationary, with no temporal falloff, we cannot use the conventional quantitative measures for QBF and can only establish its presence conclusively. We believe that these technical problems (such as wave packet construction), can be addressed straightforwardly in a more detailed analysis.

We have also briefly studied QBF for Rindler spacetimes, for which the metric allows a horizon. However the situation is qualitatively different in this case since we work in Rindler coordinates where the constant acceleration in flat Minkowski spacetime induces an effective curvature (see for example [35]). We use the same procedure as in the Schwarzschild case. However, the question of QBF across the horizon is not relevant here since Rindler coordinates are not defined beyond the Rindler wedge. We have computed the nonvanishing QBF within the Rindler wedge in Sec. IV.

The paper is organized as follows: in Sec. II, the basic formalism of constructing the Schrödinger equation for the curved spacetime Hamiltonian is elaborated. Further it is applied in the case of the Schwarzschild metric and the formal, analytic structure of the wave function is derived. In Sec. III, the observables relevant to QBF are introduced and the numerical analysis along with graphical representation of the main results are provided. This section constitutes our principal findings. In Sec. IV, we present the QBF results for Rindler spacetime along with relevant plots. Section V consists of a summary of our findings along with open future challenges. An Appendix is provided at the end showing some intermediate computational steps.

\section{SETTING UP THE SCHRÖDINGER EQUATION}

From [36] we write down the Hamiltonian in a curved background, where the $\mathrm{BH}$ metric in Cartesian coordinates reads, 


$$
\begin{aligned}
g^{00} & =\frac{1}{U} ; \quad g^{i j}=-\left[\eta^{i j}+(U-1) \frac{x^{i} x^{j}}{r^{2}}\right], \\
r^{2} & =\left(x_{1}\right)^{2}+\left(x_{2}\right)^{2}+\left(x_{3}\right)^{2}, \\
g_{00} & =U ; \quad g_{i j}=-\left[\eta_{i j}+\left(\frac{1}{U}-1\right) \frac{x_{i} x_{j}}{r^{2}}\right],
\end{aligned}
$$

with $\sqrt{-g}=1$ and $U=(1-\lambda / r)$ for the Schwarzschild metric. Following [36], $H$ is given by

$$
\begin{aligned}
H & =\left[\frac{1}{\sqrt{-g} g^{00}} \mathcal{A}+\sqrt{-g} g^{i j} \partial_{i} \partial_{j} \frac{1}{\mathcal{A}}+m^{2} \sqrt{-g} \frac{1}{\mathcal{A}}\right] \\
& =U \mathcal{A}-\left[\eta^{i j}+(U-1) \frac{x^{i} x^{j}}{r^{2}}\right] \partial_{i} \partial_{j} \frac{1}{\mathcal{A}}+m^{2} \frac{1}{\mathcal{A}}
\end{aligned}
$$

where $\mathcal{A}=\sqrt{-\nabla^{2}+m^{2}}=\sqrt{-\eta_{i j} \partial^{i} \partial^{j}+m^{2}}$. We reduce $H$ to the form,

$$
\begin{aligned}
& H=H_{0}+V ; \quad H_{0}=\mathcal{A}, \\
& V=-\frac{\lambda}{r} \mathcal{A}-\nabla^{2} \frac{1}{\mathcal{A}}+\frac{\lambda}{r^{3}} x^{i} x^{j} \partial_{i} \partial_{j} \frac{1}{\mathcal{A}}+m^{2} \frac{1}{\mathcal{A}},
\end{aligned}
$$

leading to the Schrödinger equation, $i \partial_{t} \psi-H_{0} \psi=-V \psi$,

$$
i \partial_{t} \psi-\mathcal{A} \psi=-\left[-\frac{\lambda}{r} \mathcal{A}-\nabla^{2} \frac{1}{\mathcal{A}}+\frac{\lambda}{r^{3}} x^{i} x^{j} \partial_{i} \partial_{j} \frac{1}{\mathcal{A}}+m^{2} \frac{1}{\mathcal{A}}\right] \psi .
$$

Recall that QBF in an external potential was studied in [13]. Putting back the fundamental constants, the above equation takes the form:

$$
\begin{aligned}
i \hbar \partial_{t} \psi-\sqrt{-c^{2} \hbar^{2} \nabla^{2}+c^{4} m^{2}} \psi= & -\left[\frac{2 G m}{c^{2} r} \sqrt{-c^{2} \hbar^{2} \nabla^{2}+c^{4} m^{2}}-\hbar^{2} \nabla^{2} \frac{1}{\sqrt{-c^{2} \hbar^{2} \nabla^{2}+c^{4} m^{2}}}\right. \\
& \left.+\frac{2 \hbar^{2} G M}{r^{3}} x^{i} x^{j} \partial_{i} \partial_{j} \frac{1}{\sqrt{-c^{2} \hbar^{2} \nabla^{2}+c^{4} m^{2}}}+m^{2} c^{4} \frac{1}{\sqrt{-c^{2} \hbar^{2} \nabla^{2}+c^{4} m^{2}}}\right] \psi
\end{aligned}
$$

We consider ingoing solutions along the $X$ axis as shown in Fig. 1, with a static observer outside the horizon $(r=\lambda)$ on the $X$ axis. Let us isolate the free plane wave part $\psi_{u}$ from $\psi, \psi=\psi_{u}+\psi_{s}$ with $\psi_{u}=A e^{i(-E t+\vec{k} \cdot \vec{x})}$ and subsequently

$\left[i \partial_{t} \psi_{u}-H_{0} \psi_{u}+V \psi_{u}\right]+\left[i \partial_{t} \psi_{s}-H_{0} \psi_{s}+V \psi_{s}\right]=0$

In a perturbative framework we consider exact solutions of the free equation $i \partial_{t} \psi_{u}-H_{0} \psi_{u}=0$ and subsequently calculate the first order (in $V$ ) correction $\psi_{s}$ from the equation $\left[i \partial_{t} \psi_{s}-H_{0} \psi_{s}+V \psi_{s}\right]+V \psi_{u}=0$. Now $V \psi_{u}$ acts as a source in a Greens function scheme and we drop

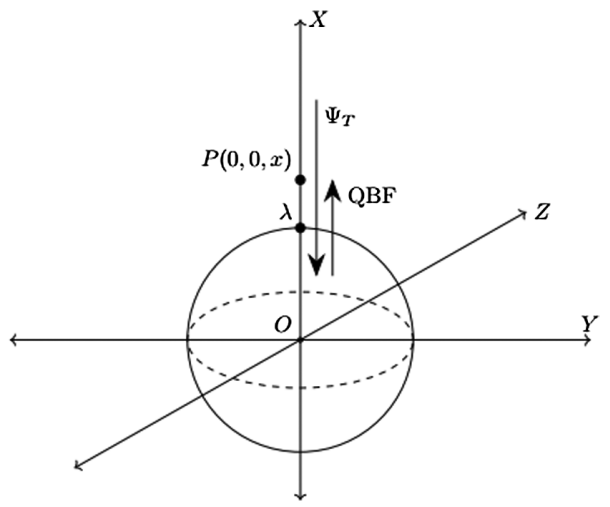

FIG. 1. $P$ denotes the observer and $\lambda$ radius of the event horizon. $\Psi_{T}(\vec{x}, t)$ is the superposed wave function going into black hole.
$V \psi_{s}$ since it will provide a correction effectively of $O\left(V^{2}\right)$ whereas we are interested in $O(V)$ corrections only. (We refer to the formalism used by Allali and Hertzberg [36].)

Using the free particle dispersion relation

$$
E_{k}-\sqrt{\hbar^{2} c^{2} k^{2}+m^{2} c^{4}}=0
$$

the equation for $\psi_{s}$ is given by

$$
\left[i \partial_{t} \psi_{s}-H_{0} \psi_{s}+V \psi_{s}\right]+V \psi_{u}=0
$$

Considering a first order potential correction for $\psi_{s}$, we drop the $V \psi_{s}$ term to obtain,

$$
i \partial_{t} \psi_{s}-H_{0} \psi_{s}+V \psi_{u}=0
$$

The computational details are provided in the Appendix. We exploit Green's function techniques to the first order, assuming the $\mathrm{BH}$ field to be weak near the horizon $(\sim \mathrm{a}$ supermassive black hole whose Schwarzschild radius exceeds its physical radius). The scattering part of $\psi$ is, therefore,

$$
\begin{aligned}
\psi_{s}^{(k)}(x, t)= & \frac{-A E_{k}}{2 \pi} e^{-i E_{k} t}\left[E_{k} \frac{e^{i k|x|}}{|x|} \int d^{3} x^{\prime}\left(1-\frac{\lambda}{\left|x^{\prime}\right|}\right) e^{i \vec{q} \cdot \vec{x}^{\prime}}\right. \\
& \left.-\frac{\lambda \hbar^{2} c^{2}}{E_{k}} k_{i} k_{j} \frac{e^{i k|x|}}{|x|} \int d^{3} x^{\prime} \frac{x^{\prime i} x^{\prime j}}{\left|x^{\prime}\right|^{3}} e^{i \vec{q} \cdot \vec{x}^{\prime}}\right]
\end{aligned}
$$


where $A$ is an arbitrary constant and $\lambda=2 G M / c^{2}$. Here, $\vec{q}=\vec{k}-k \hat{x}$. One can interpret $\vec{q}$ as a measure of the "scattering" as observed along $\hat{x}$. For, e.g., if $\vec{k}=k \hat{x}$ (the wave is directed away from the BH horizon), we expect no "scattering" to be observed. Indeed in this case, $\vec{q}=0$.

We will work with the form of $\psi^{(\vec{k})}=\psi_{u}^{(\vec{k})}+\psi_{s}^{(\vec{k})}$, where, $\psi_{u}^{(\vec{k})}=A e^{i(-E t+\vec{k} \cdot \vec{x})}$ and $\psi_{s}^{(\vec{k})}$ is given by (11).

\section{A. Working form of the wave function $\psi^{(\vec{k})}(\vec{x}, t)$}

Expressing (11) in the following form

$$
\begin{aligned}
\psi_{s}^{(\vec{k})}(\vec{x}, t)= & -\frac{A E_{k}}{2 \pi} e^{-i E_{k} t} \frac{e^{i k|\vec{x}|}}{|\vec{x}|}\left[E_{k}\left(F_{1}(\vec{q})-\lambda F_{2}(\vec{q})\right)\right. \\
& \left.+\frac{\lambda \hbar^{2} c^{2}}{E_{k}} k_{i} k_{j} \partial_{q_{i}} \partial_{q_{j}} F_{3}(\vec{q})\right],
\end{aligned}
$$

reveals three Fourier transforms,

$$
\begin{aligned}
& F_{1}(q)=\int d^{3} x^{\prime} 1 e^{i \vec{q} \cdot \vec{x}^{\prime}}=(2 \pi)^{3} \delta(\vec{q}), \\
& F_{2}(q)=\int d^{3} x^{\prime} \frac{1}{\left|x^{\prime}\right|} e^{i \vec{q} \cdot \vec{x}^{\prime}}, \quad F_{3}(q)=\int d^{3} x^{\prime} \frac{1}{\left|x^{\prime}\right|^{3}} e^{i \vec{q} \cdot \vec{x}^{\prime}} .
\end{aligned}
$$

Explicit expressions of $F_{i}(q)$ are calculated in the Appendix. Final form of the $k$ th mode of the full wave function (in first order perturbation) is

$$
\begin{aligned}
\psi^{(\vec{k})}(x, t) & =\psi_{u}^{(k)}(x, t)+\psi_{s}^{(k)}(x, t) \\
& =A e^{-i E_{k} t} e^{i(\vec{k} \cdot \vec{x})}+\psi_{s}^{(\vec{k})}(x, t) .
\end{aligned}
$$

This is one of our important results. We will use it to construct the superposition of two waves (with momenta in the same direction) to study QBF. We consider the wave just inside horizon, $|x|=\lambda-h(x)$ comprising of the ingoing modes, as is natural for a $\mathrm{BH}$, and try to ascertain QBF outside horizon, $|x|>\lambda$, as depicted in Fig. 1. The system is reduced to an effectively one (space) dimensional problem, with QBF observed at $P$ on the $X$ axis outside the horizon, $x=\lambda$.

\section{QUANTUM BACKFLOW OBSERVABLES}

QBF was observed [6] in a superposition of two plane waves with appropriate mixing coefficients and positive momentum to study the probability current $J(x, t)$. The sign of $J(x, t)$ indicates presence of QBF; for a superposition of negative momenta, the current will also be negative, at least classically. A positive current will indicate the presence of QBF. In our case, it is more convenient to calculate the current directly, following [6],

$$
J(x, t)=-i \frac{\hbar}{2 m}\left(\psi^{*}(x, t) \frac{\partial \psi(x, t)}{\partial x}-\frac{\partial \psi^{*}(x, t)}{\partial x} \psi(x, t)\right)
$$

using the form of $\psi(x, t) \equiv \psi^{(\vec{k})}(x, t)$ we have obtained.

Toy models are a convenient tool in different branches of physics, in particular when a hitherto unexplored effect is being investigated, since for the time being, showing existence of that effect is primary and quantitative estimates for possible vindication can come later. (See for example the work of Berry [14] where purely plane waves were considered in the study of QBF.) Keeping this in mind, (and following earlier works $[6,7,9,10])$, let us exploit the toy model of two-mode superposition of plane waves for QBF. The plane waves are normalized formally as Dirac deltafunction normalization or within a finite volume or with the imposition of periodic boundary condition. In existing literature in QBF simple quantum mechanical systems are considered with explicit wave packets that are localized in a finite volume. However construction of such a wave packet with finite support in our model is more difficult (which we plan to pursue in another publication). So in the present work we clearly demonstrate that it is indeed possible to have QBF from a black hole horizon but we refrain from making quantitative predictions. The only difference between our results and the QBF observables commonly used is that (since the plane waves do not diminish in magnitude) we will provide the QBF observables in local form whereas, in the latter, spatially integrated expressions are computed. Our framework, in spirit, is similar to the scattering cross section given per unit area per unit time.

\section{A. QBF from a black hole event horizon}

Let us explicitly consider $\psi^{(\vec{k})}(\vec{x}, t)=\psi_{u}^{(\vec{k})}(\vec{x}, t)+$ $\psi_{s}^{(\vec{k})}(\vec{x}, t)$ with $\psi_{u}^{(k)}(x, t)=A \exp \left(i \vec{k} \cdot \vec{x}-i E_{k} t\right)$, with $\vec{k}=$ $-k \hat{x}$ and $\vec{x}=x \hat{x}$ (position vector of $P$ in Fig. 1), leading to $\vec{q}=\vec{k}-|\vec{k}| \hat{x}=-2 k \hat{x}$.

The scattering part is given as

$$
\begin{aligned}
\psi_{s}^{(k)}= & -A \frac{E_{k}}{2 \pi} e^{-i E_{k} t} \frac{e^{i k|\vec{x}|}}{|\vec{x}|}\left[E_{k}(2 \pi)^{3} \delta(\vec{q})-E_{k} \frac{4 \pi \lambda}{q^{2}}\right. \\
& \left.+\frac{2 \pi \lambda \hbar^{2} c^{2}}{E_{k}} k^{2}\left(\lim _{\epsilon \rightarrow 0} \partial_{q_{1}}^{2}\left(-C i(q \epsilon)+\frac{2 \sin q \epsilon}{q \epsilon}\right)\right)\right], \\
= & -A \frac{E_{k}}{2 \pi} e^{-i E_{k} t} \frac{e^{i k x}}{x}\left[-E_{k} \frac{4 \pi \lambda}{4 k^{2}}+\frac{2 \pi \lambda \hbar^{2} c^{2}}{E_{k}} k^{2} \frac{q_{1}^{2}}{q^{4}}\right],
\end{aligned}
$$

with momentum $\vec{q}=q_{1} \hat{x}, \quad q^{2}=q_{1}^{2}+q_{2}^{2}+q_{3}^{2} \quad$ and $\lim _{\epsilon \rightarrow 0}\left[\partial_{q_{1}}^{2}\left(-C i(q \epsilon)+\frac{2 \sin q \epsilon}{q \epsilon}\right)\right]=-\frac{q_{2}^{2}+q_{3}^{2}-q_{1}^{2}}{q^{4}}$ simplifies to 


$$
\begin{aligned}
\psi_{s}^{(k)}(x, t) & =-\frac{A}{2 \pi} e^{-i E_{k} t} \frac{e^{i k x}}{x}\left[-\left(\hbar^{2} c^{2} k^{2}+m^{2} c^{4}\right) \frac{\pi \lambda}{k^{2}}+\frac{\pi \lambda \hbar^{2} c^{2}}{2}\right] \\
& =\frac{A}{2 \pi x} e^{-i\left(E_{k} t-k x\right)}\left[\frac{2 G M \pi}{c^{2}}\left(\frac{\hbar^{2} c^{2}}{2}+\frac{m^{2} c^{4}}{k^{2}}\right)\right] .
\end{aligned}
$$

As we have mentioned in the Introduction, there is a universal (numerical value of the) upper bound on the total amount of QBF, but the amount of QBF is different for different wave functions and one has to come up with an explicit form of wave function that generates a significant amount of QBF. In a simplified toy model approach, we have managed to find a choice of the all-important twowave superposition, $\vec{k}=-\hat{x}$ and $\vec{k}=-4 \hat{x}$, with an appreciable QBF,

$$
\begin{aligned}
\Psi_{T}(x, t)= & \psi^{(-\hat{x})}(x, t)-3 \psi^{(-4 \hat{x})}(x, t), \\
= & {\left[e^{-i\left(x-\sqrt{1+m^{2}} t\right)}-3 e^{-4 i\left(x-\sqrt{16+m^{2}} t\right)}\right] } \\
& -\frac{\pi \lambda}{2 \pi x}\left[e^{-i\left(x-\sqrt{1+m^{2}} t\right)}\left(\frac{1}{2}+m^{2}\right)-3 e^{-4 i\left(x-\sqrt{16+m^{2}} t\right)}\left(\frac{1}{2}+\frac{m^{2}}{16}\right)\right],
\end{aligned}
$$

where $\psi^{(\vec{k})}(x, t)$ is defined in (14) and we have used the calculated forms of $\psi_{u}^{(\vec{k})}(x, t)$ and $\psi_{s}^{(\vec{k})}(x, t)$. Indeed this not a unique choice and there can very well be better choices of wave functions that might generate larger QBF (within the upper bound). To show that QBF generating superpositions are quite easily available, we have provided two specific examples of different superposition along with their respective QBF, at the end of this section. However, one needs to search for the particular wave function that induces the maximum amount of QBF.

For a general wave superposition of the form

$$
\psi(x)=\rho(x) e^{i \int_{0}^{x} d x^{\prime} k\left(x^{\prime}\right)}
$$

the amplitude function $\rho(x)$ and local wave number function $k(x)$

$$
k(x)=\frac{d}{d x} \arg \psi(x)=\operatorname{Im} \frac{(d \psi(x) / d x)}{\psi(x)}
$$

are real. Positive and negative $k(x)$ will indicate that the wave is traveling in positive or negative direction at $x$. Applying this definition (see for example $[1,6,14]$ for more details) to a superposition of plane waves with positive momenta (for example)

$$
\psi(x)=\sum_{n=0}^{N} C_{n} e^{i k_{n} x} ; \quad k_{n} \geq 0
$$

one can show that $k(x)$ can have negative values. As a specific example $[1,6,14]$ for

$$
\psi(x)=1-a e^{i x}
$$

the local wave number $k(x)$, given by

$$
k(x)=a \frac{a-\cos x}{1+a^{2}-2 a \cos x},
$$

will generate QBF for $a<1$ within $|x|<\arccos (a)$ periodically.

\section{B. Numerical analysis}

The analytic expressions of the probability and current densities, $\zeta(x, t)=\Psi_{T}^{*}(x, t) \Psi_{T}(x, t)$ and $J(x, t)$ are, respectively, given by

$$
\begin{aligned}
\zeta(x, t)= & \frac{1}{1024 x^{2}}\left[5\left(2048 x^{2}-64\left(16+5 m^{2}\right) x \lambda+\left(128+80 m^{2}+53 m^{4}\right) \lambda^{2}\right)-48\left(128 x^{2}-4\left(16+17 m^{2}\right) x \lambda\right.\right. \\
& \left.\left.+\left(8+17 m^{2}+2 m^{4}\right) \lambda^{2} t\right) \cos \left(\left(\sqrt{1+m^{2}}-\sqrt{16+m^{2}}\right) t-3 x\right)\right], \\
J(x, t)= & \frac{\hbar}{\left(256 m x^{2}\right)}\left[-9472 x^{2}+4736 x \lambda+832 m^{2} x \lambda-592 \lambda^{2}-208 m^{2} \lambda^{2}-73 m^{4} \lambda^{2}+30\left(128 x^{2}-4\left(16+17 m^{2}\right) x \lambda\right.\right. \\
& \left.\left.+\left(8+17 m^{2}+2 m^{4}\right) \lambda^{2}\right) \cos \left[\left(\sqrt{1+m^{2}}-\sqrt{16+m^{2}}\right) t-3 x\right]+360 m^{2} \lambda \sin \left[\sqrt{1+m^{2}} t-\sqrt{16+m^{2}} t-3 x\right]\right] .
\end{aligned}
$$

The functions are analytic, $\forall x \neq 0, \forall t \geq 0$; i.e., it is nonanalytic only at the curvature singularity at $x=0$ and not at the coordinate singularity at $x=\lambda$. In Fig. 2 we show the variation in probability density for two values of $\lambda$. There appears to be a qualitative change in the behavior after the horizon, that is more pronounced for smaller $\lambda$. 
$\lambda=20$

$\zeta(x, t=0)$
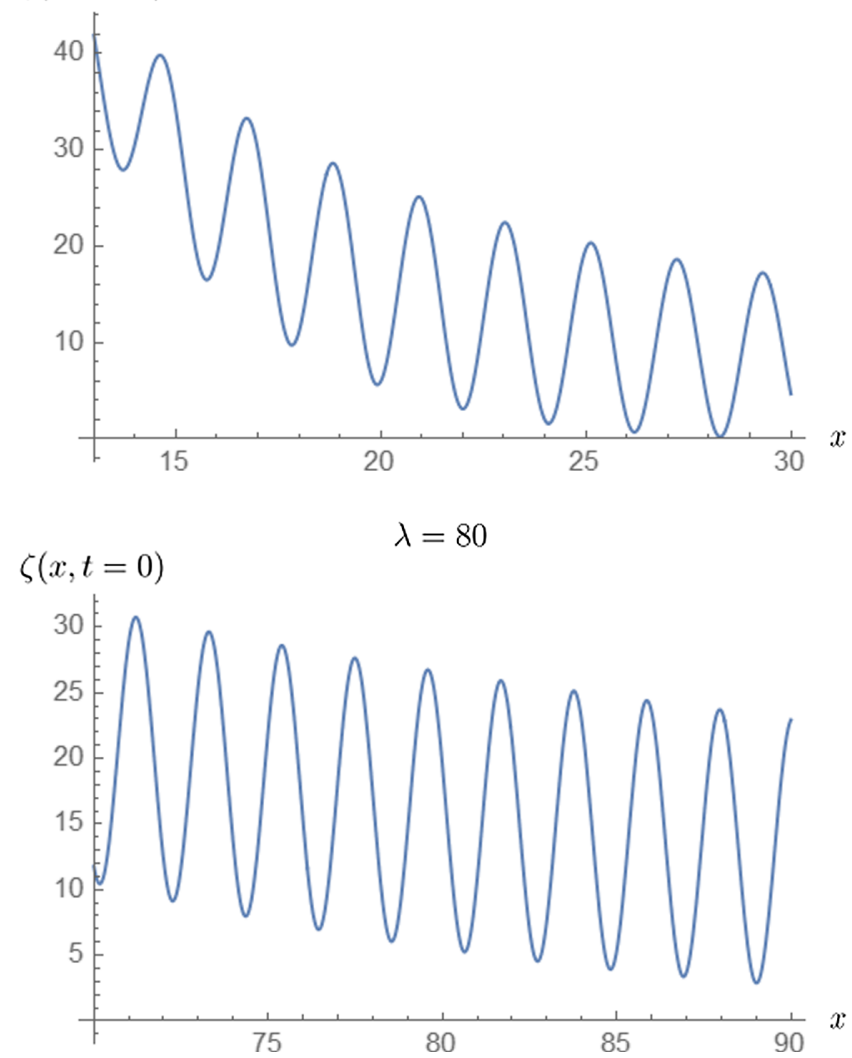

FIG. 2. Plots of the density function $\zeta(x, t=0)$ for two values of $\lambda$.

In Fig. 3, snapshots of the density profile are shown for two different times. Notice that the density is greater inside the horizon and as expected, falls off away from the horizon, on the outside. The time-stationary behavior is readily visible.

Our most decisive results appear in the profile in Fig. 4, where we plot the current $J(x, t)$ for $\hbar=1, m=3$, for two values of $\lambda$. In Fig. 4, we see that there is a small QBF region for $t=0$ (actually for all values of $t$ as well) near the horizon $x=\lambda$, that is shaded red in the figure. It can be shown numerically that $J(x, t)$ is strictly negative (as expected naïvely) for $x \ll \lambda$ as well as for $x \gg \lambda$. This can also be seen from the time slices in Fig. 4. The envelope of the oscillatory plots in Fig. 4 is only positive for a finite region surrounding $x=\lambda$ as shown. This suggests that QBF is originated from only a finite region around the Schwarzchild BH event horizon. A different superposition and its associated QBF is provided in Fig. 5.

In fact size and shape of the region from where QBF emerges changes (periodically) with time. Such a shift in the QBF peaks is depicted for two different times $t=0$ and $t=5$, in Fig. 6, an enlarged view of the QBF zone (marked in red) for the $\lambda=20$ case of Fig. 4 . In Fig. 4 it is to be understood that $\mathrm{QBF}$ regions occur periodically along the intervals in $x$. In Fig. 6 the QBF zone at $t=0$ (marked in $\lambda=60$

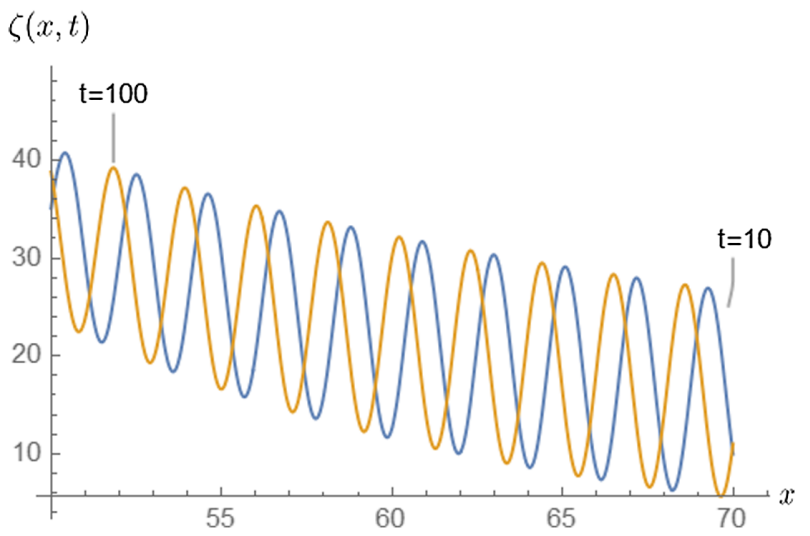

FIG. 3. Plot of $\zeta(x, t)$ at two different times, $t=10$ and $t=100$, for $\lambda=60$.

$$
\lambda=20
$$
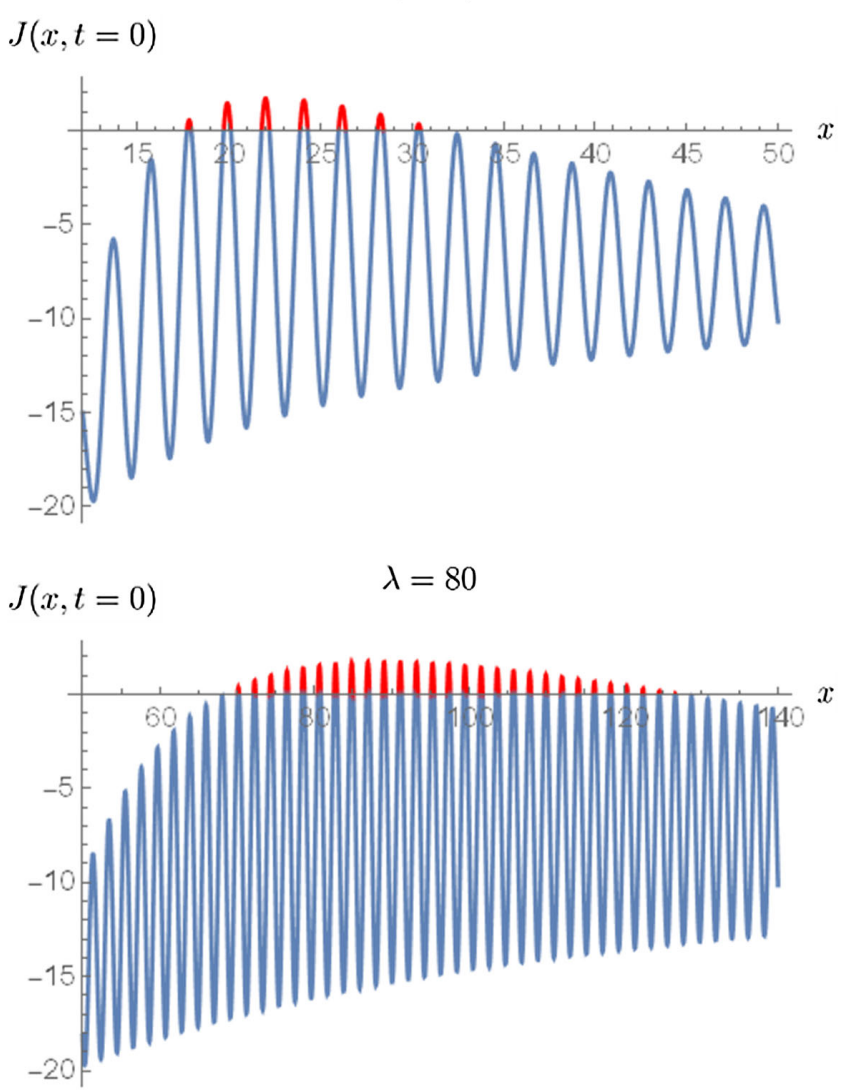

FIG. 4. QBF (red region) observed from the plots of $J(x, t=0)$ for two values of $\lambda$, on superposing $\vec{k}=-\hat{x}$ and $\vec{k}=-4 \hat{x}$ solutions. The size of the QBF region increases with $\lambda$.

blue) are different from the QBF zones at $t=5$ (marked in yellow) indicating how the QBF zones evolve with time. Note that only for plane wave superpositions the corresponding QBF occur periodically. Similar situations in other QBF models are discussed in detail in [14]. 


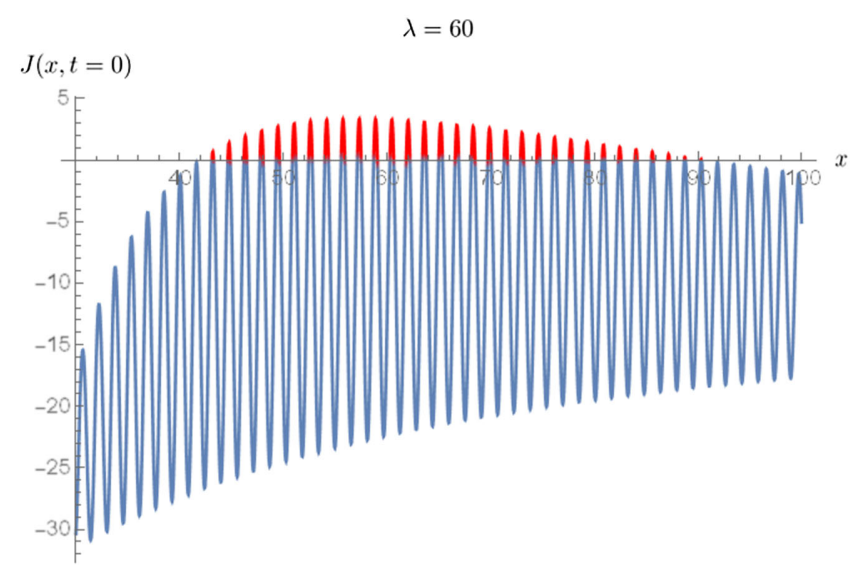

FIG. 5. $J(x, t=0)$ plot showing sizeable QBF on superposing solutions with $\vec{k}=-\hat{x}$ and $\vec{k}=-5 \hat{x}$.

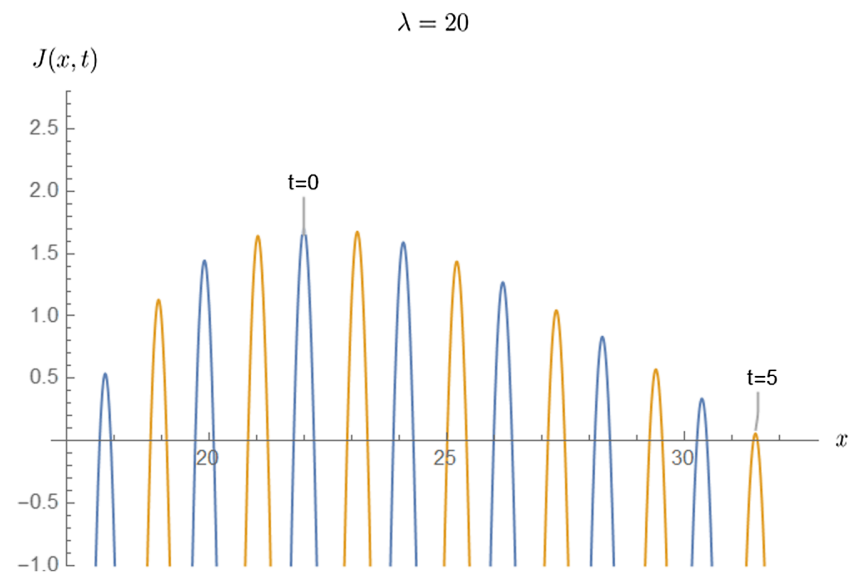

FIG. 6. A magnified plot of the QBF region for $t=0$ (blue curve) and $t=5$ (yellow curve), showing evolution of the QBF region with time.

\section{QUANTUM BACK FLOW FOR RINDLER OBSERVERS}

In this section we will briefly present another example of QBF for a spacetime that has a close connection with the Schwarzschild spacetime but has also essential differences that is reflected in the corresponding QBF; the Rindler spacetime (see for example [35]). Quite interestingly, even though Rindler metric has a horizon, because of the qualitative difference in the nature of the two horizons the $\mathrm{QBF}$ also has a different structure that we will elaborate.

The Rindler horizon appears in the following way. The world line of a point particle moving with constant proper acceleration (that is, the acceleration measured by an observer comoving with the said point) in flat $(1+1)$ dimensional spacetime will be a hyperbola. World lines of light rays asymptote to the arms of the hyperbola. Hence the light ray paths constitute horizons bounding the Rindler wedge in the sense that any signal from outside the wedge cannot reach the accelerating particle since to do that it has to overtake the light pulse. On the other hand, from the point of view of the accelerated observer, the light pulse is always at a constant distance ( $\sim$ inverse of the proper acceleration) behind.

Clearly, the Rindler horizon is different from a Schwarzschild black hole horizon: the former will be present so long as the acceleration persists and is not eternal as the latter (in the classical sense, disregarding Hawking radiation). Furthermore the true singular nature of the latter metric, where the curvature is infinite, is absent in the former. Still, the horizon analogy can be pursued further: in the Rindler case, a constant force is required to maintain a constant proper distance from the Rindler horizon; in the Schwarzschild case, a (redshifted) force ( $\sim$ surface gravity) on a particle is needed to keep it stationary on the horizon. Again, considering a sufficiently massive black hole (so that the spacetime curvature is extremely small), similar phenomena (red shift, time dilation and so on) occur in the vicinity of both the horizons and Unruh radiation in Rindler is an analog of Hawking radiation in Schwarzschild. With an appropriate rescaling, the Rindler metric structurally reduces to a Schwarzschild form.

A convenient way to study the effects of a constant acceleration is to exploit Rindler coordinates. For acceleration along the direction of motion, it is possible to show that the acceleration is the same for an intertial frame and in the proper frame of the accelerating particle. Hence one can solve the motion of a particle in a Minkowski (laboratory) frame in a straightforward way and transform it to the comoving frame in which the accelerating particle is at rest, which in this case is the Rindler frame. Interestingly the metric in Rindler frame is that of a curved spacetime, with a horizon, the Rindler horizon. This is agreeable to us since we have already analyzed QBF for a generic curved spacetime and can simply borrow the machinery to compute QBF for Rindler spacetime. However, since the Rindler metric is only defined within the Rindler wedge, it is not feasible to consider QBF across the Rindler horizon. One might heuristically argue that QBF supposedly cannot violate special relativity, which would have been the case if QBF across a Rindler horizon was allowed. However, as we demonstrate below, the good news is that QBF is nonvanishing inside the Rindler wedge.

As explained just above, the solution of Newton's dynamical equation,

$$
\begin{aligned}
m a & =\frac{d P}{d T}=m \frac{d(\gamma U)}{d T}, \quad U=d X / d T, \\
\gamma & =1 /\left(\sqrt{1-U^{2} / c^{2}}\right),
\end{aligned}
$$

with $a$, the proper acceleration of the observer, being given by 


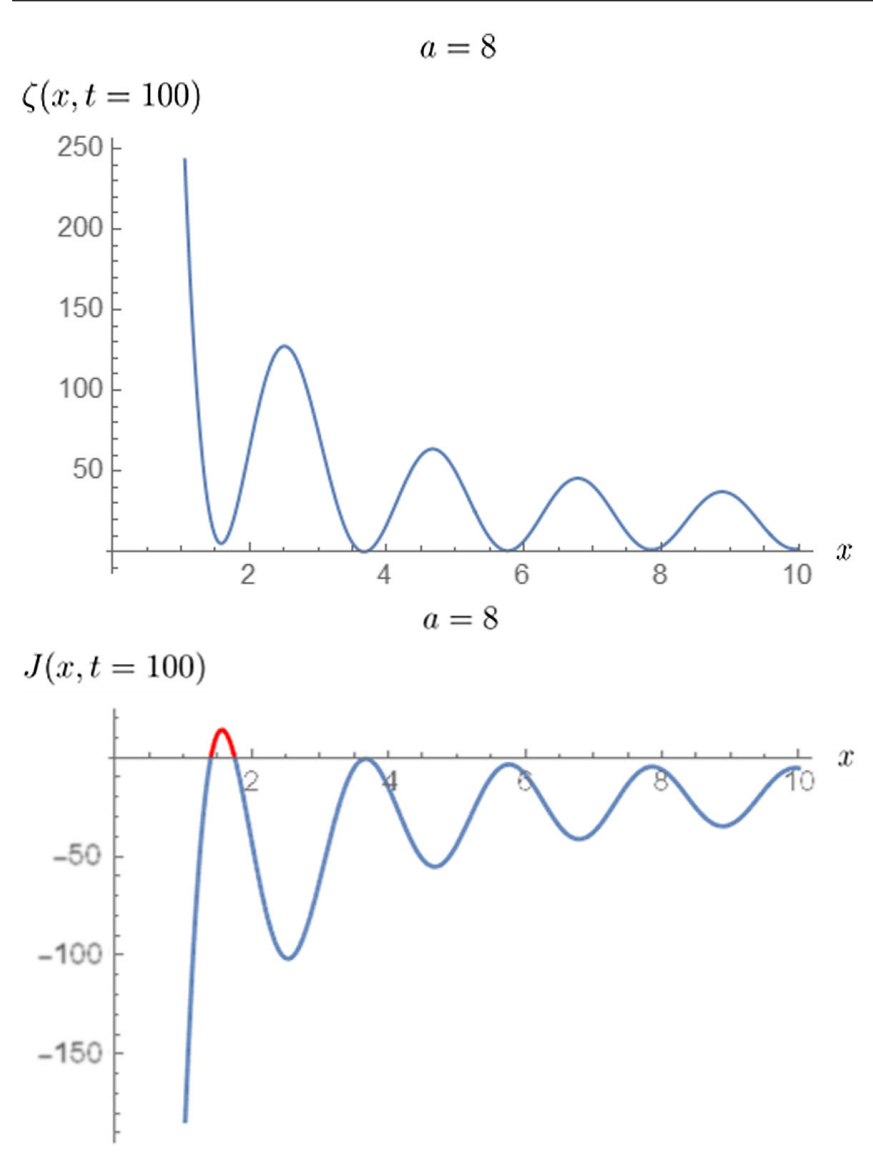

FIG. 7. Plots of the density function $\zeta(x, t=100)$ (top) and the current $J(x, t=100)$ for $|x| \in\left(\frac{1}{a}, 10\right)$.

$$
U=a T /\left(\sqrt{1+(a T / c)^{2}}\right) .
$$

Defining the proper time $t$ by $d T / d t=1 /\left(\sqrt{1-U^{2} / c^{2}}\right)$ one obtains the transformation laws [35]

$$
\begin{aligned}
& X=\left(x+\frac{1}{a}\right) \sinh \left[a\left(t-t_{0}\right)\right]+X_{0}-\frac{1}{a}, \\
& T=\left(x+\frac{1}{a}\right) \cosh \left[a\left(t-t_{0}\right)\right]+T_{0},
\end{aligned}
$$

connecting the flat Minkowski metric

$$
d s^{2}=-d T^{2}+d X^{2}
$$

to the Rindler metric (in terms of proper time and space coordinates $x, t$ ) given by

$$
d s^{2}=-(1+x a) d t^{2}+d x^{2},
$$

where we have used the $(-1,+1)$ signature and $c=1$. Clearly, for $x \in \mathbb{R} \backslash\{-1 / a\}$, the Minkowski coordinates satisfy $-T<X<T$ (the Rindler wedge). Following the same procedure outlined in the next two sections, we obtain the scattering part of the wave function as, $\psi_{s}^{\operatorname{Rind}(k)}(t, \vec{x})=A \frac{E_{k}}{2 \pi|\vec{x}|} e^{-i\left(E_{k} t-k \mid \vec{x}\right) \mid}\left[\frac{4 a}{q^{2}}-2 \pi \delta(\vec{q})\right]$. We construct the superposition of two wave functions, with $\vec{k}=-\hat{x}$ and $\vec{k}=-4 \hat{x}$ and plot the probability density $\zeta(x, t)$ and current density $J(x, t)$ in Fig. 7. From the plot of $J(x, t)$ in Fig. 7, we see that QBF indeed occurs (marked in red) away from the Rindler horizon at $|x|=\frac{1}{a}$; i.e., QBF is observed strictly "inside" the Rindler wedge. Note that there are no divergences in the two profiles of Fig. 7 near the horizon; we had to scale it up so that the small amount of QBF shows up clearly, leading to large but finite values of the respective observables near horizon.

\section{DISCUSSION}

To summarize, we have studied the QBF across the event horizon of a Schwarzschild $\mathrm{BH}$, using perturbative solutions of the Schrödinger equation for a particle in the $\mathrm{BH}$ background, near the horizon. In a superposition of two ingoing modes, QBF is observed across the horizon. Interestingly QBF persists for a finite spatial range. In our stationary model, overall time dependence and related observations of charge and current density are uniform (without any decay). In the present toy model approach, we have not attempted to provide quantitative estimates in terms of a conventional observable, the total probability (integrated over position) of QBF. We believe this weakness can be overcome by considering wave packets, instead of a simple superposition, as done here. However it should be noted that wave propagation and wave packet construction in curved (space and) spacetime is itself an involved problem that has created a lot of interest in recent years as well; some of the novel features related to curved spacetime being decoherence of wave packet due to its interaction with zero-point fluctuations produced by a gravitational wave, ambiguity in defining a particle excitation, among others (some relevant references are [37-41]). Keeping this in mind we have adhered to the toy model presented here which can establish presence of a QBF across BH horizon. We plan to introduce wave packets to study the QBF in this context in near future.

We have also provided a brief description of $\mathrm{QBF}$ in Rindler space where it occurs inside the Rindler wedge.

The present work raises several open questions and possibilities: how do we interpret the $\mathrm{QBF}$ across $\mathrm{BH}$ horizon? Can the QBF correspond to outgoing modes? Does there exist any form of correlation between the ingoing and outgoing (QBF) part of the wave function? Even though QBF is an effect, distinct from information leakage through Hawking radiation, can it lead to information exchange between inside and outside horizon? Can this type of QBF be realized in analog gravity models? We conclude by noting several major differences between QBF 
(in $\mathrm{BH}$ case) and Hawking effect: QBF is a generic quantum process present in wave function evolution, while the Hawking effect is a semiclassical field theoretic phenomenon applicable near the event horizon of a $\mathrm{BH}$. QBF is intimately connected to the external matter wave function moving across $\mathrm{BH}$ horizon, whereas no external matter degrees of freedom are involved in Hawking effect. Again, there is no BH mass depletion due to QBF (it can only acquire the mass of the ingoing part of wave function). Also QBF is active for a limited period of time (for wave packets as ingoing modes), and is restricted in a spatial domain whereas $\mathrm{BH}$ mass decreases via Hawking radiation resulting in a $\mathrm{BH}$ evaporation. The latter is present throughout the time the $\mathrm{BH}$ is alive.

Since we have mooted the question of a possible connection between QBF and information leakage, it is natural to ask whether there are any comparable time scales involved in the two processes. One such scale for black holes is the scrambling time proposed in [42] that refers to the process of quick delocalization of quantum information in thermal states. It scales as the logarithm of the number of degrees of freedom of the system. It was also hypothesized that "Black holes are the fastest scramblers in nature" [42]. Before proceeding further let us observe that in the Schwarzschild black hole case that we have discussed in detail, we have considered time independent solutions and thus analysis of time scales is beyond the scope of the present work. But, in previous works involving time dependent QBF $[6,7,10]$ it has been shown that generically QBF persists for a finite period of time and it can be nonzero for several time periods. However one should be cautious since the time period can be scaled to different durations with the restriction that the integrated amount of probability transfer by QBF remains the same $[6,7,10]$. Indeed, more study is needed before one can make any conjecture regarding a possible connection between the time scales involved in QBF and information scrambling time (or any other relevant time scale) for black holes.

Open problems (i) use renormalizable ingoing wave packets for quantitative estimates of QBF, and (ii) investigate $\mathrm{QBF}$ in analog gravity models. As a physically interesting example, in coastal region where a river empties into an ocean (depending critically upon external parameters), a tidal bore, i.e., a strong tidal wave, can occur that pushes up the river, against the current. In the horizon analogy for a tidal bore, a smooth wave front is followed by a series of waves, traveling upstream forced by high tides [43-45]. The bore acts as a horizon, separating an analog white hole up along the river. There is no wave propagation upstream to the bore and only rapidly decaying evanescent waves can exist there in the analogue white hole region. If we push the identification between QBF and tidal bore further, then in the white hole situation the leakage of the evanescent waves might be interpreted as the QBF. Recall that QBF also remains active for a finite period of time (provided we use wave packets for the ingoing modes). Clearly this analogy demands further study.

We end by noting that a deeper analysis of the physical interpretation of the QBF part is needed since apart from an intriguing remark by Berry [11] regarding possible particlelike nature of the $\mathrm{QBF}$ sector, not many observations are present in the literature.

\section{ACKNOWLEDGMENTS}

We thank Bibhas Ranjan Majhi for suggestions in the early stage of the work. We are also grateful to Professor Michael Berry and Professor Arseni Goussev for correspondence. We also thank Professor Nava Gaddam and Professor Merab Gogberashvili for informing us of relevant references. We are thankful to the referees for constructive comments.

\section{APPENDIX}

In the Appendix we have elucidated some parts of our calculations.

Equation (11) of main text.- We provide details of the derivation of the solution of Schrödinger equation, using Green's function [36]

$\psi_{s}^{(\vec{k})}(x, t)=\int d^{4} x^{\prime} G_{4}\left(t-t^{\prime} ; x-x^{\prime}\right) V\left(t^{\prime}, x^{\prime}\right) \psi_{u}^{(\vec{k})}\left(t^{\prime}, x^{\prime}\right)$,

which can be rewritten as

$$
\begin{aligned}
\psi_{s}^{(k)}(x, t) & =e^{-i E_{k} t} \int d^{3} x^{\prime} G_{3}\left(x-x^{\prime}\right) V\left(x^{\prime}\right) \psi_{u}^{(\vec{k})}\left(x^{\prime}\right), \\
& =e^{-i E_{k} t} \int d^{3} x^{\prime} \frac{-E_{k}}{2 \pi\left|x-x^{\prime}\right|} e^{i k\left|x-x^{\prime}\right|}\left[-\frac{2 G M}{c^{2}\left|x^{\prime}\right|} \mathcal{A}-\hbar^{2} \nabla^{2} \frac{1}{\mathcal{A}}+\frac{2 \hbar^{2} G M}{\left|x^{\prime}\right|^{3}} x^{\prime i} x^{\prime j} \partial_{i}^{\prime} \partial_{j}^{\prime} \frac{1}{\mathcal{A}}+m^{2} c^{4} \frac{1}{\mathcal{A}}\right]\left(A e^{i k \cdot x^{\prime}}\right),
\end{aligned}
$$


where $E_{k}^{2}=\hbar^{2} c^{2} k^{2}+m^{2} c^{4}$. In the above, $G_{4}\left(t-t^{\prime} ; x-x^{\prime}\right)$ and $G_{3}\left(x-x^{\prime}\right)$ refer to the four- and three-dimensional Green's functions, respectively. We use the notation of [36]. This yields

$$
\begin{aligned}
\psi_{s}^{(k)}(x, t)= & \frac{-A E_{k}}{2 \pi} e^{-i E_{k} t} \int d^{3} x^{\prime} \frac{e^{i k\left|x-x^{\prime}\right|}}{\left|x-x^{\prime}\right|}\left[-\frac{\lambda}{\left|x^{\prime}\right|} \sqrt{k^{2}+m^{2}}\right. \\
& +k^{2} \frac{1}{\sqrt{k^{2}+m^{2}}}+m^{2} \frac{1}{\sqrt{k^{2}+m^{2}}} \\
& \left.-\frac{\lambda}{\left|x^{\prime}\right|^{3}} x^{\prime i} x^{\prime j} k_{i} k_{j} \frac{1}{\sqrt{k^{2}+m^{2}}}\right]\left(e^{i k . x^{\prime}}\right) . \quad \text { (A3) }
\end{aligned}
$$

After simplification we find

$$
\begin{aligned}
\psi_{s}^{(k)}(x, t)= & \frac{-A E_{k}}{2 \pi} e^{-i E_{k} t} \int d^{3} x^{\prime} \frac{e^{i k\left|x-x^{\prime}\right|}}{\left|x-x^{\prime}\right|} \\
& \times\left[\left(1-\frac{2 G M}{c^{2}\left|x^{\prime}\right|}\right) E_{k}-\frac{2 G M}{E_{k}} \hbar k_{i} \hbar k_{j} \frac{x^{\prime i} x^{\prime j}}{\left|x^{\prime}\right|^{3}}\right]\left(e^{i k . x^{\prime}}\right) .
\end{aligned}
$$

We concentrate on the region near horizon $|x|=\lambda \gg\left|x^{\prime}\right|$ and expand

$$
\rightarrow\left|x-x^{\prime}\right| \approx|x|-\frac{\vec{x} \cdot \vec{x}^{\prime}}{|x|} \equiv|x| \approx \lambda-\hat{x} \cdot \vec{x}^{\prime}
$$

so that in a standard approximation scheme in (A4),

$$
\begin{aligned}
\frac{e^{i k\left|x-x^{\prime}\right|}}{\left|x-x^{\prime}\right|} e^{i k \cdot x^{\prime}} & \approx \frac{e^{i k\left(|x|-\hat{x} \cdot \vec{x}^{\prime}\right)}}{|x|-\hat{x} \cdot \vec{x}^{\prime}} e^{i k \cdot x^{\prime}} \approx \frac{e^{i k|x|}}{|x|} e^{i(\vec{k}-k \hat{x}) \cdot \vec{x}^{\prime}} \\
& =\frac{e^{i k|x|}}{|x|} e^{i \vec{q} \cdot \vec{x}^{\prime}} ; \quad \vec{q}=\vec{k}-k \hat{x} .
\end{aligned}
$$

Substituting these relations in (A4) we recover Eq. (11) of original text.

Equation (13) of main text and $F_{i}(q)$.- Exploiting spherical symmetry for a generic case yields

$$
F(q)=\frac{4 \pi}{q} \int_{0}^{\infty} d r V(r) r \sin (q r) .
$$

For $V=1 / r$, a regularization (in the form of a mass scale $\mu$ ) is needed:

$$
F_{2}(q ; \mu)=\frac{4 \pi}{q} \int_{0}^{\infty} d r \frac{e^{-\mu r}}{r} r \sin (q r)=\frac{4 \pi}{\mu^{2}+q^{2}},
$$

where $\vec{q}=\vec{k}-k \hat{r} ; q=2 k \sin (\theta / 2)$. Finally taking $\mu \rightarrow 0$ we find

$$
F_{2}(q)=\frac{4 \pi}{q^{2}}
$$

On the other hand, for $F_{3}(q)$ we find, using the same prescription as above, that

$$
F_{3}(q)=\frac{4 \pi}{q} \int_{0}^{\infty} \frac{\sin (q r)}{r^{2}} d r
$$

diverges due to the singularity at $r=0$. Therefore, we perform the integral,

$$
\bar{F}_{3}(q)=\frac{4 \pi}{q} \int_{\epsilon}^{\infty} \frac{\sin (q r)}{r^{2}} d r .
$$

for some, $\epsilon \in \mathbb{R}^{+}$.

Then we have

$\bar{F}_{3}(q)=2 \pi\left(-C i(q \epsilon)+2 \ln (q)-\ln \left(q^{2}\right)+2 \frac{\sin (q \epsilon)}{q \epsilon}\right)$,

where, $\operatorname{Ci}(z)$ is the cosine integral function defined as

$$
C i(z)=-\int_{z}^{\infty} \frac{\cos t}{t} d t
$$

whose series expansion about 0 , is given as, $C i(x)=\gamma+\ln (x)+\sum_{k=1}^{\infty} \frac{\left(-x^{2}\right)^{k}}{2 k(2 k) !}$. Here $\gamma$ is the EulerMascheroni constant.

The value of $2 \ln (q)-\ln \left(q^{2}\right)$ is $2 \pi i$ if $q<0$ and 0 if $q>0$. Thus, more compactly,

$\bar{F}_{3}(q)=2 \pi\left(-C i(q \epsilon)+2 \pi i \Theta(-q)+2 \frac{\sin (q \epsilon)}{q \epsilon}\right)$,

where $\Theta(x)$ denotes the Heaviside-Theta function. $F_{3}(\vec{q})$ will be approximated by $\bar{F}_{3}(\vec{q})$ and the limit $\epsilon \rightarrow 0$ will be taken after differentiating $\bar{F}_{3}(\vec{q})$.

One-dimensional result (17) with units:

$\psi_{s}^{(k)}(x, t)=\frac{A}{2 \pi x} e^{-i\left(E_{k} t-k x\right)}\left[\frac{2 G M \pi}{c^{2}}\left(\frac{1}{2}+\frac{m^{2} c^{2}}{\hbar^{2} k^{2}}\right)\right]$. 
[1] G. R. Allcock, Ann. Phys. (N.Y.) 53, 253 (1969); 53, 311 (1969).

[2] J. G. Muga, J. P. Palao, and C. R. Leavens, Phys. Lett. A 253, 21 (1999).

[3] J. G. Muga and C. R. Leavens, Phys. Rep. 338, 353 (2000).

[4] J. Kijowski, Rep. Math. Phys. 6, 361 (1974).

[5] W. van Dijk and F. M. Toyama, Phys. Rev. A 100, 052101 (2019).

[6] A. J. Bracken and G. F. Melloy, J. Phys. A 27, 2197 (1994).

[7] J. M. Yearsley, J. J. Halliwell, R. Hartshorn, and A. Whitby, Phys. Rev. A 86, 042116 (2012).

[8] M. Penz, G. Grubl, S. Kreidl, and P. Wagner, J. Phys. A 39, 423 (2006).

[9] J. J. Halliwell, E. Gillman, O. Lennon, M. Patel, and I. Rami, J. Phys. A 46, 475303 (2013).

[10] J. M. Yearsley and J. J. Halliwell, J. Phys. Conf. Ser. 442, 012055 (2013).

[11] M. V. Berry, J. Phys. A 45, 185308 (2012).

[12] J. Ashfaque, J. Lynch, and P. Strange, Phys. Scr. 94, 125107 (2019).

[13] G. F Melloy and A. J. Bracken, Ann. Phys. (Leipzig) 7, 726 (1998).

[14] M. V. Berry, J. Phys. A 43, 415302 (2010).

[15] A. Goussev, Phys. Rev. A 99, 043626 (2019).

[16] Y. Eliezer, T. Zacharias, and A. Bahabad, Optica 7, 72 (2020).

[17] M. Palmero, E. Torrontegui, J. G. Muga, and M. Modugno, Phys. Rev. A 87, 053618 (2013).

[18] Sh. Mardonov, M. Palmero, M. Modugno, E. Ya. Sherman, and J. G. Muga, Europhys. Lett. 106, 60004 (2014).

[19] M. Miller, W. C. Yuan, R. Dumke, and T. Paterek, Quantum 5, 379 (2021).

[20] M. Barbier and A. Goussev, Quantum 5, 536 (2021).

[21] A. Goussev, Phys. Rev. A 103, 022217 (2021).

[22] S. Hawking, Phys. Rev. D 14, 2460 (1976).
[23] S. Hawking, Commun. Math. Phys. 43, 199 (1975).

[24] J. D. Bekenstein, Phys. Rev. D 7, 2333 (1973).

[25] D. N. Page, Phys. Rev. Lett. 71, 1291 (1993).

[26] G. Penington, J. High Energy Phys. 09 (2020) 002.

[27] A. Almheiri, N. Engelhardt, D. Marolf, and H. Maxfield, J. High Energy Phys. 12 (2019) 063.

[28] A. Almheiri, T. Hartman, J. Maldacena, E. Shaghoulian, and A. Tajdini, Rev. Mod. Phys. 93, 035002 (2021).

[29] G. Hooft, Int. J. Mod. Phys. A 11, 4623 (1996).

[30] G. Hooft, Found. Phys. 46, 1185 (2016).

[31] N. Gaddam, N. Groenenboom, and G. Hooft, arXiv: 2012.02357.

[32] P. Betzios, N. Gaddam, and O. Papadoulaki, J. High Energy Phys. 07 (2021) 017.

[33] M. Gogberashvili, Int. J. Theor. Phys. 57, 1763 (2018).

[34] M. Gogberashvili, Int. J. Theor. Phys. 58, 3711 (2019).

[35] B. Knorr, Uniform Relativistic Acceleration, http://www .physik.uni-leipzig.de (2010).

[36] I. Allali and M. P. Hertzberg, J. Cosmol. Astropart. Phys. 07 (2020) 056.

[37] W. L. Power and I. C. Percival, Proc. R. Soc. A 456, 955 (2000).

[38] M. Arminjon and F. Reifler, J. Phys. 306, 012061 (2011).

[39] V. A. Emelyanov, Eur. Phys. J. C 81, 189 (2021).

[40] T. Masaaki, M. Y. Ishizuka, and W. A. Okosa, Prog. Theor. Phys. 84, 5 (1990).

[41] A. Patsyk, M. A. Bandres, R. Bekenstein, and M. Segev, Phys. Rev. X 8, 011001 (2018).

[42] Y. Sekino and L. Susskind, J. High Energy Phys. 10 (2008) 065.

[43] M. V. Berry, New J. Phys. 20, 053066 (2018).

[44] M. V. Berry, New J. Phys. 21, 073021 (2019).

[45] We are thankful to Professor Michael Berry for pointing out the possibility of this analogy. 